\title{
CARPAL TUNNEL SYNDROME AMONG SYMPTOMATIC SCHOOL TEACHERS ATTENDING UNIVERSITY HOSPITAL
}

By

Younis $\mathrm{F}^{1}$, El-batanony $\mathrm{M}^{1,2}$ and Fotoh $\mathrm{D}^{3}$

${ }^{1}$ Department of Public Health and Community Medicine, ${ }^{3}$ Department of Physical Medicine, Rheumatology and Rehabilitation, Faculty of Medicine, Menoufia University, Menoufia, Egypt, ${ }^{2}$ Unaizah College of Medicine, Qassim University, Saudi Arabia Corresponding author: Younis F: faten.younis@med.menofia.edu.eg

\begin{abstract}
Introduction: School teachers are prone to various occupational problems especially musculoskeletal disorders including carpal tunnel syndrome (CTS) which may cause sick leave, absenteeism and early retirement leading to decreased productivity at work. In developing countries, a paucity of researches was done in this field. Aim of work: To assess the prevalence and the risk factors of CTS among symptomatic school teachers in Menoufia, Egypt. Materials and methods: A cross-sectional study of symptomatic school teachers at the Physical Medicine, Rheumatology and Rehabilitation Department, Menoufia University Hospital from January to October 2018. A selfadministered questionnaire including participants' personal, occupational and pain information were used. Also, nerve conduction studies were done of both right and left median nerves including motor and sensory. Results: One hundred and eighty seven school teachers responded to the study. The prevalence of CTS was reported by $62 \%$ of symptomatic teacher. Both hands of CTS teachers had significantly higher mean latency and lower amplitude and nerve conduction velocity of motor and sensory nerve studies when compared to non CTS one. The severity of carpal tunnel syndrome in both hands of CTS teachers was mainly moderate grade. The CTS was significantly prevalent among female school teachers and primary or secondary level of school. A combination of risk factors: body mass index, school level, working duration, working hours per day and working computer hours per day; were found to be significantly associated with increasing CTS. Conclusion and recommendations: The symptomatic school teachers had high prevalence of CTS which is affecting their work and education system as a whole. An implement intervention programs is needed by the higher authorities to address this issue. An adequate care, ergonomic rules and exercise program have to be recommended so as to alleviate this disorder.
\end{abstract}

Key words: Carpal Tunnel Syndrome, School teachers, Risk factors and Nerve conduction. 


\section{Introduction}

Musculoskeletal disorders (MSD) among school teachers are numerous. They include many inflammatory and degenerative conditions affecting the muscles, peripheral nerves (carpal tunnel syndrome, sciatica.....), tendons (tenosynovitis), bone, joint and ligaments. The condition may be caused or aggravated by work tasks (Erick and Smith, 2015).

The common risk factors for MSD among teachers were prolonged desk work, prolonged standing in the class and repetitive overhead writing on the board, prolonged sitting resulting from frequent reading, preparation of lessons and marking of assignments and working on a computer (Shuai et al., 2014).

Carpal tunnel syndrome (CTS) is a symptomatic compressive median nerve neuropathy as it passes in the carpal tunnel; characterized by hand pain, numbness, and tingling through median nerve distribution (thumb, index, middle finger, and the radial side of the ring finger). There are several causes for CTS such as hypothyroidism, diabetes, and rheumatoid arthritis. In late pregnancy CTS may occur but usually transient (Harris-Adamson et al., 2015).
There is a strong association between development of CTS among teachers and different individual, ergonomic, and occupational factors as body mass index (BMI), female gender, repeated flexion and extension of the wrist, repetitive movements of the wrist for more than 30 seconds and total work time more than $50 \%$ in repetitive movement patterns (Boschman et al., 2015).

A clear history and targeted examination, which identifies standard features and provocative factors, increases the likelihood for diagnosis of CTS (American Academy of Orthopaedic Surgeons, 2007). Nerve conduction studies can be used in patients with an intermediate pretest probability or in patients with an atypical presentation. They can also be used to quantify and stratify disease severity to aid in further treatment decision (Burton et al., 2014).

Different types of musculoskeletal disorders including CTS decrease work productivity and one of the leading cause for ill health retirement (Mengestu, 2013). Based on our knowledge, many studied were done in Egypt on MSD of teachers not focused on CTS, so this study is intended to be performed. 


\section{Aim of work}

To assess the prevalence and the risk factors of Carpel Tunnel Syndrome among symptomatic school teachers in Menoufia, Egypt.

\section{Materials and methods:}

Study design: It is a cross-sectional study.

Place and duration of the study: this study was carried out during the period from the $1^{\text {st }}$ of January to the last of October, 2018 at the Physical Medicine, Rheumatology and Rehabilitation Department, Menoufia University Hospital, Egypt.

Study sample: All symptomatic teachers attending the previously mentioned departments during the period of the study were included. The teachers of various school levels (primary, preparatory and secondary), all ages, both sex and those had work experience more than one year were recruited.

Exclusion criteria: Musculoskeletal health problems, neurological problems, acute or post-acute illnesses, metabolic disorders, pregnant teachers and less than one year of experience. One hundred ninety six teachers attended to the Physical Medicine, Rheumatology and Rehabilitation department during the mentioned duration of the study. The eligible participated teachers after application exclusion criteria were 187 with response rate $95.4 \%$.

Study methods:

1- A predesigned questionnaire was conducted. It included information on personal and occupational histories (work duration, working hours per day and computer hours per day), general health status, general use of the wrist and hands as well as symptoms related to CTS.

Questions that were asked for a patient presenting with hand or wrist symptoms:

1. Do you have numbness or tingling in your wrist, hand, or fingers?

2. Do your symptoms spare your little finger?

3. Are the symptoms worse at night?

4. Do the symptoms wake you up at night?

5. Have you noticed your hand is weak; for example, have you found yourself dropping things?

6. Do you find shaking your hand, holding your hand or running it under warm water improves your symptoms?

7. Are the symptoms made worse by activities such as driving, holding a telephone, using vibrating tools, or typing? 
8. Have splints or injections helped with your pain if you have had it in the past?

\section{2- Clinical examination was done} to all participants.

Assessment was done for: weakness/atrophy of thenar muscle (Werner, 2006) as well as sensory abnormality (Graham et al., 2006). Tinel's Sign in which tap on the median nerve with a reflex hammer were carried out. If there is an electric-like sensation, the test is positive. Moreover, the Phalen's Maneuver or the wrist-flexion test in which the patient presses the backs of physician hands and fingers together with physician wrists flexed and fingers pointed down and stay for 1-2 minutes (Burton et al., 2013 and 2014).

Based on the previously reported manifestations, the diagnosis was: (1) nocturnal or activity-related pain or dysesthesia limited to the hand; (2) sensory deficit or reduced two-point discrimination in the median nerve distribution; (3) isolated atrophy of the abductor policies brevis muscle (APB); and (4) positive Phalen's or Tinel's signs. The diagnosis of CTS was suspected when the patients complained of painful dysesthesia in the sensory area of the median nerve and one of the criteria (2-4) was fulfilled (Lee et al., 2011).

\section{3- Investigations:}

Nerve Conduction Velocity Test (Electrophysiological Studies) was applied to the studied group. Nerve conduction studies of both right and left median nerves including motor and sensory studies were performed. All patients were studied with multiple electro diagnostic studies (EDX) for CTS as follows (Wang and Yan, 2013): all latencies except median distal motor latency (MDL) were measured to the negative peak. The amplitude was measured from the baseline to the peak of negative deflection. All tests were using the same electromyography (EMG)/nerve conduction velocity (NCV) instrument with a percutaneous supramaximal response. The pulse duration was $0.05 / 0.1$ millisecond for sensory and mixed nerve stimulation and 0.2/0.5 millisecond for motor nerve stimulation. The filters were set at 20 $\mathrm{Hz}$ and $2 \mathrm{kHz}$. The sweep speed was set at 1 millisecond per division. The onecentimeter disc recordings were used for mixed nerve studies and ring electrodes for sensory studies. A ground electrode was appropriately placed between the stimulating and recording electrodes.

a) Sensory Nerve Conduction Studies: Using antidromic techniques 
with stimulation applied to the wrist, median sensory nerve action potentials can be recorded at the thumb, the index, the long, and the ring fingers. We used a fixed $13 \mathrm{~cm}$ distance from the ring recording electrode (G1) that was placed on the mid portion of the proximal phalanx, and a reference ring electrode (G2) that was placed on the mid portion of the middle phalanx of the same finger, $3 \mathrm{~cm}$ from the active recording (G1) electrode. The cutoff value was 3.5 milliseconds.

b) Motor Nerve Conduction Studies: Obtained recording over the abductor policies brevis (APB) muscle. We used a fixed $7 \mathrm{~cm}$ distance from the disc recording electrodes that were placed over the belly of the abductor policies brevis (APB) muscle (the recording electrode) and just distal to the metacarpophalangeal joint (the reference electrode). The cutoff value was 4.4 milliseconds.

Diagnosis of CTS, in the present study, was based on the characteristic hand symptoms and the results of clinical examination as well as nerve conduction studies. Cherian and Kuruvilla, 2006 were classified CTS into five grades as grade 1 (very mild), grade 2 (mild), grade 3 (moderate), grade 4 (severe) and grade 5 (extreme).

\section{Consent}

A written consent was obtained from all participants. The purpose of the study was explained to the examined teachers and assured privacy. Confidentiality was maintained.

\section{Ethical Approval}

The study protocol was approved by the Committee for Medical Research Ethics at Menoufia Faculty of Medicine.

\section{Data management}

Data were analyzed using the IBM Statistical Package for Social Sciences (SPSS) statistics version 20 (SPSS Inc., Chicago, IL). Participants were classified into two groups regarding diagnosis of CTS (CTS group and non-CTS one). Chi-square test was used to examine the relation between qualitative variables. For quantitative data, comparison between two groups was done using either student t-test or Mann-Whitney test (non-parametric t-test) as appropriate. Binary logistic regression, Odds ratio (OR) with its $95 \%$ confidence interval (CI) were used for risk estimation. Spearman-rho method was used to test the correlation between numerical variables. A p-value $\leq 0.05$ was considered statistically significant. 


\section{Results}

Table (1): Socio-demographic characteristics of the studied group.

\begin{tabular}{|c|c|c|c|}
\hline \multirow[t]{2}{*}{$\begin{array}{l}\text { Socio-demographic } \\
\text { characteristics }\end{array}$} & \multicolumn{2}{|c|}{$\begin{array}{c}\text { Studied symptomatic } \\
\text { teachers }\left(\mathrm{No}_{0}=187\right)\end{array}$} & \multirow[t]{2}{*}{$p$ value } \\
\hline & $\begin{array}{l}\text { CTS cases } \\
(\text { No }=116)\end{array}$ & $\begin{array}{c}\text { Non CTS } \\
(\text { No }=71)\end{array}$ & \\
\hline $\begin{array}{l}\text { Age (years) } \\
\quad<35 \\
\quad \geq 35\end{array}$ & $\begin{array}{ll}57 & 49.1 \% \\
59 & 50.9 \%\end{array}$ & $\begin{array}{ll}29 & 40.8 \% \\
42 & 59.2 \%\end{array}$ & 0.30 \\
\hline $\begin{array}{l}\text { Gender } \\
\text { Male } \\
\text { Female } \\
\end{array}$ & $\begin{array}{ll}17 & 14.7 \% \\
99 & 85.3 \% \\
\end{array}$ & $\begin{array}{ll}38 & 53.5 \% \\
33 & 46.5 \% \\
\end{array}$ & $<0.001 * *$ \\
\hline $\begin{array}{l}\text { Working in which } \\
\text { school level } \\
\text { Primary } \\
\text { Preparatory } \\
\text { Secondary }\end{array}$ & $\begin{array}{ll}17 & 14.7 \% \\
39 & 33.6 \% \\
60 & 51.7 \%\end{array}$ & $\begin{array}{cc}30 & 42.2 \% \\
32 & 45.1 \% \\
9 & 12.7 \%\end{array}$ & $\begin{array}{c}<0.001 * * \\
0.12 \\
<0.001 * *\end{array}$ \\
\hline $\begin{array}{c}\text { BMI }\left(\mathbf{K g} / \mathbf{m}^{2}\right) \\
\text { Mean } \pm \text { SD } \\
\end{array}$ & $29.7 \pm 5.6$ & $25.1 \pm 4.7$ & $<0.001 * *$ \\
\hline $\begin{array}{l}\text { Working duration } \\
\text { (years) } \\
\text { Mean } \pm \text { SD }\end{array}$ & $10.1 \pm 6.8$ & $7.3 \pm 4.5$ & $0.001 * *$ \\
\hline $\begin{array}{l}\text { Working hours / day } \\
\text { Mean } \pm \text { SD }\end{array}$ & $6.8 \pm 1.9$ & $5.6 \pm 2.2$ & $<0.001 * *$ \\
\hline $\begin{array}{l}\text { Working hours with } \\
\text { computer / day } \\
\text { Mean } \pm \text { SD }\end{array}$ & $4.03 \pm 0.85$ & $2.9 \pm 1.7$ & $<0.001 * *$ \\
\hline
\end{tabular}

**: Highly statistically significant difference

CTS: Carpal Tunnel Syndrome

BMI: Body Mass Index

Table 1 showed that $50.9 \%$ who was complaining of CTS were $\geq 35$ of age, the majority of them were female $(85.3 \%)$ and $51.7 \%$ were working in a secondary schools. There was a highly statistically significant difference between the mean of BMI, working duration (years), working hours per day, working hours with computer per day and CTS. 
Figure (1): Clinical manifestations of the studied teachers regarding gender

\section{Clinical manifestations of studied teachers regarding gender}

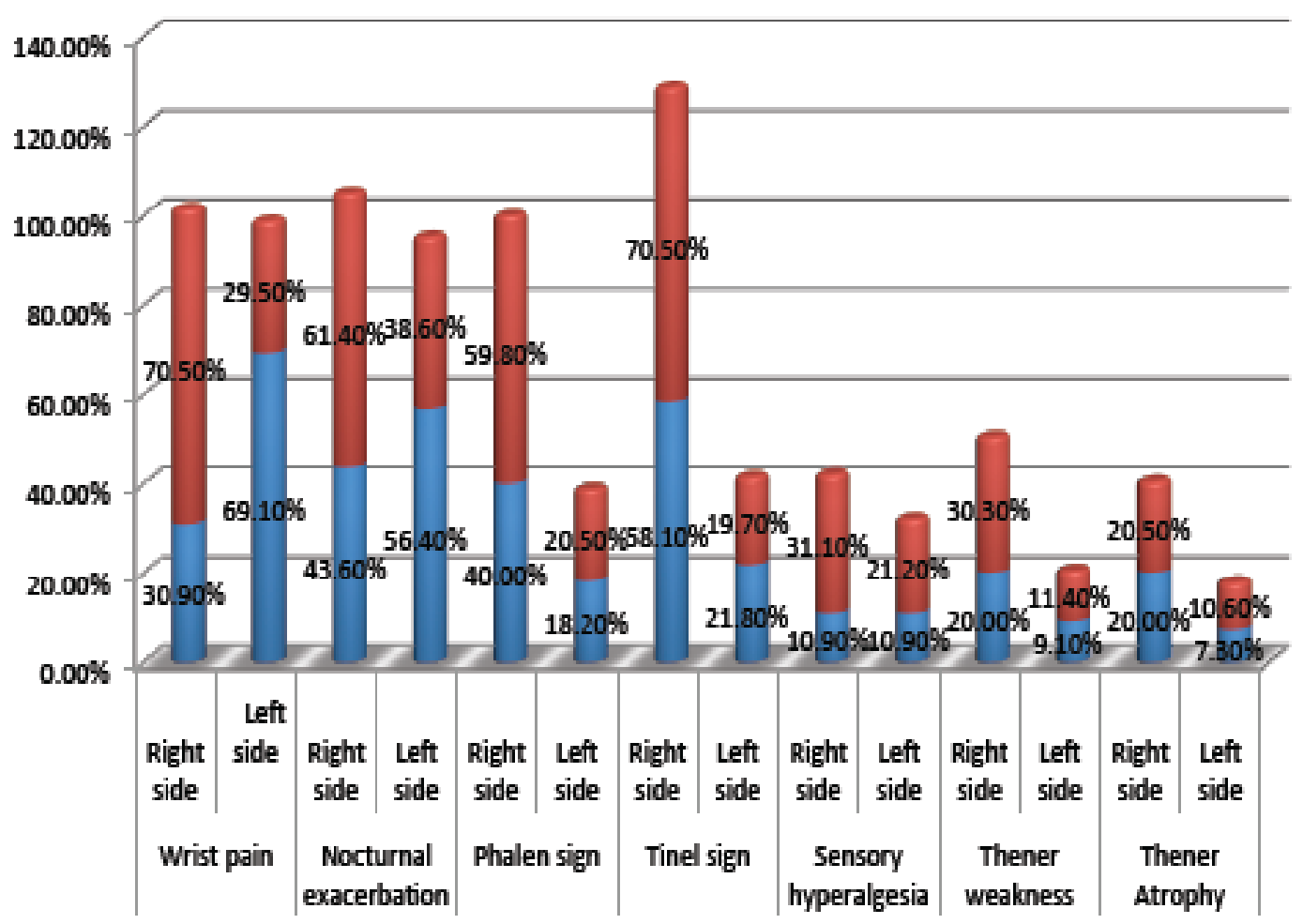

Male teachers $\quad$ Female teachers

Figure (1) showed that the most prevalent manifestations on both hands of studied male and female teachers were wrist pain, nocturnal exacerbation and positive Tinel's or Phalen's signs. Female teachers had high prevalence of these manifestations for the right hand than male teachers $(\mathrm{p}<0.05)$. 
Table (2): Motor and sensory nerve conduction study of the examined teachers.

\begin{tabular}{|c|c|c|c|c|}
\hline \multirow{2}{*}{\multicolumn{2}{|c|}{$\begin{array}{l}\text { Nerve conduction study } \\
\text { CTS cases } \\
(\mathbf{N o}=\mathbf{1 1 6})\end{array}$}} & \multicolumn{2}{|c|}{ Studied teachers } & \multirow{2}{*}{ p value } \\
\hline & & \multirow{2}{*}{$\begin{array}{c}\begin{array}{c}\text { Non CTS } \\
(\text { No }=71)\end{array} \\
7.5 \pm 2.7 \\
7.8 \pm 2.7 \\
\end{array}$} & \multirow[b]{2}{*}{$\begin{array}{l}6.2 \pm 2.1 \\
4.7 \pm 1.9\end{array}$} & \\
\hline \multirow{3}{*}{ 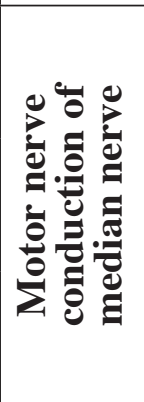 } & $\begin{array}{l}\text { Latency }(\text { Mean } \pm \text { SD ) } \\
\text { Right side } \\
\text { Left side }\end{array}$ & & & $\begin{array}{c}0.02 * \\
<0.001 * *\end{array}$ \\
\hline & $\begin{array}{l}\text { Amplitude (Mean } \pm \text { SD) } \\
\text { Right side } \\
\text { Left side }\end{array}$ & $\begin{array}{l}4.4 \pm 3.9 \\
7.8 \pm 3.0\end{array}$ & $\begin{array}{l}8.1 \pm 3.1 \\
7.8 \pm 2.4\end{array}$ & $\begin{array}{c}<\mathbf{0 . 0 0 1} * * \\
0.45\end{array}$ \\
\hline & $\begin{array}{l}\text { NCV (Mean } \pm \text { SD) } \\
\text { Right side } \\
\text { Left side }\end{array}$ & $\begin{array}{l}50.4 \pm 7.2 \\
51.8 \pm 7.3\end{array}$ & $\begin{array}{c}63.9 \pm 11.1 \\
57.3 \pm 8.5\end{array}$ & $\begin{array}{c}<0.001 * * \\
0.003 *\end{array}$ \\
\hline \multirow{3}{*}{ 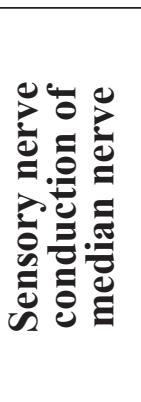 } & $\begin{array}{l}\text { Latency }(\text { Mean } \pm \text { SD }) \\
\text { Right side } \\
\text { Left side }\end{array}$ & $\begin{array}{l}3.9 \pm 1.5 \\
4.1 \pm 1.5\end{array}$ & $\begin{array}{l}1.6 \pm 0.42 \\
2.7 \pm 0.61\end{array}$ & $\begin{array}{l}<0.001 * * \\
<0.001 * *\end{array}$ \\
\hline & $\begin{array}{l}\text { Amplitude }(\text { Mean } \pm \text { SD }) \\
\text { Right side } \\
\text { Left side }\end{array}$ & $\begin{array}{c}12.3 \pm 3.9 \\
8.8 \pm 5.9 \\
\end{array}$ & $\begin{array}{l}22.1 \pm 7.5 \\
18.4 \pm 7.0 \\
\end{array}$ & $\begin{array}{l}<0.001 * * \\
<0.001 * *\end{array}$ \\
\hline & $\begin{array}{l}\text { NCV (Mean } \pm \text { SD ) } \\
\text { Right side } \\
\text { Left side }\end{array}$ & $\begin{array}{l}43.9 \pm 7.5 \\
44.7 \pm 7.6 \\
\end{array}$ & $\begin{array}{c}54.5 \pm 10.6 \\
50.3 \pm 9.7 \\
\end{array}$ & $\begin{array}{c}<0.001 * * \\
0.002 *\end{array}$ \\
\hline \multirow{3}{*}{ 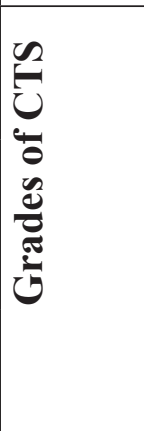 } & $\begin{array}{l}\text { Mild CTS (No \& \%) } \\
\text { Right side } \\
\text { Left side }\end{array}$ & \multicolumn{3}{|c|}{$\begin{array}{l}22.2 \% \\
27.4 \% \\
\end{array}$} \\
\hline & $\begin{array}{l}\text { Moderate CTS (No \& } \\
\text { \%) } \\
\text { Right side } \\
\text { Left side }\end{array}$ & \multicolumn{3}{|c|}{$\begin{array}{l}34 \\
35\end{array}$} \\
\hline & $\begin{array}{l}\text { Severe CTS (No \& \%) } \\
\text { Right side } \\
\text { Left side }\end{array}$ & \multicolumn{2}{|r|}{$\begin{array}{r}8 \\
10\end{array}$} & \\
\hline
\end{tabular}

Table (2) showed that there were statistically significant higher mean latency and lower amplitude and nerve conduction velocity of motor and sensory nerve studies among CTS teachers in both hands when compared to non CTS one $(\mathrm{P}<0.05)$. The severity of CTS in both hands among teachers was mainly moderate grade (63\% and $56.5 \%$ for right and left hands; respectively). 


\section{Table (3): Spearman's correlation between results of nerve conduction study and risk factors among the studied teachers}

\begin{tabular}{|c|c|c|c|c|c|c|c|}
\hline \multirow[b]{2}{*}{ Right side } & \multicolumn{3}{|c|}{ Motor nerve conduction } & \multicolumn{3}{|c|}{ Sensory nerve conduction } & \multirow[t]{2}{*}{ Diagnosis grade } \\
\hline & Latency & Amplitude & NCV & Latency & Amplitude & NCV & \\
\hline \begin{tabular}{|l|} 
Work \\
duration \\
(years)
\end{tabular} & $\begin{array}{l}r=0.277 \\
\mathbf{p}=\mathbf{0 . 0 0 7} *\end{array}$ & $\begin{array}{c}r=-0.207 \\
\mathbf{p}=\mathbf{0 . 0 4} *\end{array}$ & $\begin{array}{c}\mathrm{r}=0.040 \\
\mathrm{p}=0.70\end{array}$ & $\begin{array}{c}r=330 \\
\mathbf{p}=\mathbf{0 . 0 2} *\end{array}$ & $\begin{array}{l}r=-0.310 \\
\mathbf{p}=\mathbf{0 . 0 0 2} *\end{array}$ & $\begin{array}{c}\mathrm{r}=0.188 \\
\mathrm{p}=0.07\end{array}$ & $\begin{array}{c}r=0.310 \\
\mathbf{p}=\mathbf{0 . 0 0 2} *\end{array}$ \\
\hline $\begin{array}{l}\text { Working } \\
\text { hours per } \\
\text { day }\end{array}$ & $\begin{array}{c}r=0.200 \\
p=0.06\end{array}$ & $\begin{array}{c}\mathrm{r}=-0.092 \\
\mathrm{p}=0.37\end{array}$ & $\begin{array}{l}\mathrm{r}=0.025 \\
\mathrm{p}=0.81\end{array}$ & $\begin{array}{l}r=0.239 \\
\mathbf{p}=\mathbf{0 . 0 2} *\end{array}$ & $\begin{array}{c}r=-270 \\
\mathbf{p}=\mathbf{0 . 0 0 8} *\end{array}$ & $\begin{array}{c}r=-0.144 \\
p=0.16\end{array}$ & $\begin{array}{l}\mathrm{r}=0.258 \\
\mathbf{p}=\mathbf{0 . 0 1} *\end{array}$ \\
\hline \begin{tabular}{|l|} 
Working \\
hours with \\
computer \\
per day
\end{tabular} & $\begin{array}{c}\mathrm{r}=0.097 \\
\mathrm{p}=0.35\end{array}$ & $\begin{array}{c}\mathrm{r}=-0.078 \\
\mathrm{p}=0.45\end{array}$ & $\begin{array}{c}\mathrm{r}=0.136 \\
\mathrm{p}=0.19\end{array}$ & $\begin{array}{l}r=0.267 \\
\mathbf{p}=\mathbf{0 . 0 0 9} *\end{array}$ & $\begin{array}{c}\mathrm{r}=0.089 \\
\mathrm{p}=0.39\end{array}$ & $\begin{array}{c}\mathrm{r}=-0.031 \\
\mathrm{p}=0.77\end{array}$ & $\begin{array}{c}\mathrm{r}=0.334 \\
\mathbf{p}=\mathbf{0 . 0 0 1} * *\end{array}$ \\
\hline BMI & $\begin{array}{c}r=0.173 \\
p=0.09\end{array}$ & $\begin{array}{c}\mathrm{r}=-0.274 \\
\mathrm{p}=0.14\end{array}$ & $\begin{array}{c}\mathrm{r}=0.132 \\
\mathrm{p}=0.07\end{array}$ & $\begin{array}{c}r=0.518 \\
p=0.64\end{array}$ & $\begin{array}{c}r=-0.174 \\
\mathbf{p}=\mathbf{0 . 0 3} *\end{array}$ & $\begin{array}{c}\mathrm{r}=0.132 \\
\mathrm{p}=0.07\end{array}$ & $\begin{array}{c}r=0.152 \\
\mathbf{p}=\mathbf{0 . 0 4} *\end{array}$ \\
\hline \multirow[b]{2}{*}{ Left side } & \multicolumn{3}{|c|}{ Motor nerve conduction } & \multicolumn{3}{|c|}{ Sensory nerve conduction } & Diagnosis grade \\
\hline & Latency & Amplitude & NCV & Latency & Amplitude & NCV & \\
\hline $\begin{array}{l}\text { Work } \\
\text { duration } \\
\text { (years) }\end{array}$ & $\begin{array}{r}r=0.098 \\
p=0.35\end{array}$ & $\begin{array}{c}\mathrm{r}=0.120 \\
\mathrm{p}=0.25\end{array}$ & $\begin{array}{c}\mathrm{r}=0.005 \\
\mathrm{p}=0.96\end{array}$ & $\begin{array}{c}\mathrm{r}=0.096 \\
\mathrm{p}=0.36\end{array}$ & $\begin{array}{c}r=-0.209 \\
\mathbf{p}=\mathbf{0 . 0 4} *\end{array}$ & $\begin{array}{c}\mathrm{r}=0.177 \\
\mathrm{p}=0.09\end{array}$ & $\begin{array}{c}r=0.318 \\
\mathbf{p}=\mathbf{0 . 0 0 2} *\end{array}$ \\
\hline \begin{tabular}{|l|} 
Working \\
hours per \\
day
\end{tabular} & $\begin{array}{c}r=0.195 \\
p=0.06\end{array}$ & $\begin{array}{c}r=-0.118 \\
p=0.26\end{array}$ & $\begin{array}{c}\mathrm{r}=-0.117 \\
\mathrm{p}=0.27\end{array}$ & $\begin{array}{c}\mathrm{r}=0.133 \\
\mathrm{p}=0.21\end{array}$ & $\begin{array}{c}\mathrm{r}=-0.090 \\
\mathrm{p}=0.39\end{array}$ & $\begin{array}{c}\mathrm{r}=-0.109 \\
\mathrm{p}=0.29\end{array}$ & $\begin{array}{l}r=0.254 \\
\mathbf{p}=\mathbf{0 . 0 1} *\end{array}$ \\
\hline \begin{tabular}{|l|} 
Working \\
hours with \\
computer \\
per day \\
\end{tabular} & $\begin{array}{c}r=0.286 \\
\mathbf{p}=\mathbf{0 . 0 0 6 *}\end{array}$ & $\begin{array}{c}r=-0.045 \\
p=0.67\end{array}$ & $\begin{array}{c}\mathrm{r}=0.141 \\
\mathrm{p}=0.18\end{array}$ & $\begin{array}{l}r=0.261 \\
\mathbf{p}=\mathbf{0 . 0 1} *\end{array}$ & $\begin{array}{c}\mathrm{r}=-0.253 \\
\mathrm{p}=0.01\end{array}$ & $\begin{array}{c}r=-0.033 \\
p=0.75\end{array}$ & $\begin{array}{c}r=0.420 \\
\mathbf{p}<\mathbf{0 . 0 0 1} * *\end{array}$ \\
\hline \begin{tabular}{|l|l} 
BMI \\
\end{tabular} & $\begin{array}{c}r=0.309 \\
p=0.09\end{array}$ & $\begin{array}{c}r=-0.191 \\
p=0.08\end{array}$ & $\begin{array}{c}\mathrm{r}=0.132 \\
\mathrm{p}=0.32\end{array}$ & $\begin{array}{c}\mathrm{r}=0.318 \\
\mathrm{p}=0.72\end{array}$ & $\begin{array}{c}r=-0.487 \\
p=0.07\end{array}$ & $\begin{array}{c}r=0.117 \\
\mathrm{p}=0.18\end{array}$ & $\begin{array}{c}r=0.138 \\
\mathbf{p}=\mathbf{0 . 0 5} *\end{array}$ \\
\hline
\end{tabular}

*: Statistically significant difference

$\mathrm{NCV}=$ Nerve conduction velocity

BMI: Body Mass Index

Table (3) showed that a statistically significant difference between the latency of sensory and motor nerve fibers as well as severity of CTS grade in both hands and the years of work duration, working hours/day, while the amplitude decreased $(\mathrm{p}<0.05)$. 
Table (4): Binary logistic regression analysis to detect risk factors of CTS.

\begin{tabular}{|c|c|c|c|c|}
\hline Risk factors & B & $\begin{array}{l}\text { Odds } \\
\text { ratio }\end{array}$ & p value & $95 \% \mathrm{CI}$ \\
\hline $\begin{array}{l}\text { Gender } \\
\text { Male } \\
\text { Female } \\
\end{array}$ & 1.63 & 4.11 & $0.001 * *$ & $\begin{array}{c}1 \\
1.71-9.88 \\
\end{array}$ \\
\hline $\mathbf{B M I}\left(\mathrm{Kg} / \mathrm{m}^{2}\right)$ & 0.29 & 1.49 & $0.01 *$ & $1.03-2.15$ \\
\hline Working duration /years & 0.12 & 2.63 & 0.004* & $2.12-3.26$ \\
\hline Working hours / day & 0.21 & 1.99 & $0.002 *$ & $1.45-2.73$ \\
\hline $\begin{array}{l}\text { Working hours with } \\
\text { computer / day }\end{array}$ & 0.21 & 1.23 & $0.03 *$ & $1.02-1.48$ \\
\hline $\begin{array}{l}\text { Working in which school } \\
\text { level }\end{array}$ & & & & \\
\hline $\begin{array}{l}\text { Primary } \\
\text { Preparatory } \\
\text { Secondary }\end{array}$ & $\begin{array}{l}0.05 \\
0.63 \\
0.44\end{array}$ & $\begin{array}{l}0.98 \\
1.94 \\
2.63\end{array}$ & $\begin{array}{c}0.22 \\
\mathbf{0 . 0 3} * \\
\mathbf{0 . 0 0 4} *\end{array}$ & $\begin{array}{l}0.91-1.06 \\
1.13-3.33 \\
2.04-3.39\end{array}$ \\
\hline
\end{tabular}

*: Statistically significant difference

**: Highly statistically significant difference

Table (4) showed that among the studied personal and occupational risk factors, the following had a significant association with CTS among teachers: being female $(\mathrm{OR}=4.11 ; 95 \% \mathrm{CI}: 1.71-9.88)$, preparatory $(\mathrm{OR}=1.94 ; 95 \% \mathrm{CI}: 1.13-3.33)$ and secondary school teachers $(\mathrm{OR}=2.63 ; 95 \% \mathrm{CI}: 2.04-3.39)$, with increasing BMI $(\mathrm{OR}=1.49 ; 95 \% \mathrm{CI}: 1.03-2.15)$, work duration years $(\mathrm{OR}=2.63$; 95\% CI:2.12-3.26), hours of working per day $(\mathrm{OR}=1.99 ; 95 \% \mathrm{CI}: 1.45-2.73)$ and working hours with the computer per day $(\mathrm{OR}=1.23 ; 95 \% \mathrm{CI}: 1.02-1.48)(\mathrm{p}<0.05)$. 


\section{Discussion}

The present study aimed to determine the prevalence of CTS among symptomatic teachers at Menoufia Governorate, Egypt and to assess the different risk factors as personal and occupational ones. Clinical examinations and electrophysiological studies were evaluated among all participated teachers for diagnosis CTS.

The CTS prevalence among the studied symptomatic teachers was $62 \%$ (116/187) (Table 1). To our knowledge, the prevalence of CTS documented by previous studies was done among different occupations rather than teachers while many past researches were performed to assess wrist/hand pain among teachers in Turkey, Australia and India $(13.4 \%, 30.7 \%$ and $15.75 \%$; respectively) (Korkmaz et al., 2011 - Erick and Smith, 2014 - Nirav and Sanket, 2018). As the studied participants were symptomatic teachers, the present study had the high prevalence of wrist/hand pain.

In the current study, being a female was at increasing risk of CTS than males. There was a highly statistically significant difference between working duration (years), working hours per day, working hours with computer per day and CTS (Table 1). This was in agreement with what was reported by Erick and Smith, 2014 on their study on musculoskeletal disorders among teaching profession in developing countries. Also this work was in accordance with what concluded by Cardoso et al., 2009 in Brazil on their study among teachers.

The current study was in contrast with another study done by Alsiddiky et al., 2014 on their work on the prevalence of musculoskeletal pain and its associated factors among female Saudi school teachers and found no significant correlation with the number of teaching hours, and musculoskeletal pain.

Wrist pain, nocturnal exacerbation and positive Tinel and Phalen signs were the highest prevalent manifestations among teachers in the current study especially female teachers when compared to male (Figure 1). This came in agreement with previous researches (Cardoso et al., 2009, Korkmaz et al., 2011 and Erick and Smith, 2014).

The current study reported a significant difference between CTS teachers and non CTS ones as regard the median nerve motor and sensory conduction studies. The main CTS grade of this study was a moderate grade of both hand sides (Table 2). Also, Yacoub, 2002 in his study on carpal tunnel syndrome recorded that $70 \%$ of the hands with electrophysiological CTS were moderate and severe. 
This present work showed that there was a statistically significant difference between the latency of sensory and motor nerve fibers as well as the severity of CTS grade in both hands and the years of work duration, working hours/day, while the amplitude decreased $(\mathrm{p}<0.05)$ (Table 3). Erick and Smith, 2014 in their study on the prevalence and risk factors for musculoskeletal disorders among school teachers in Botswana detected that the longer employment duration, the higher the chance of getting job-related disorders among teachers.

Another aim of this study was to determine the risk factors associated with CTS teachers. Logistic regression analysis revealed a number of interesting associations between CTS and personal and occupational factors. Of the personal factors, female gender and increasing BMI were positively associated with the development of CTS. Female teachers were four folds in comparison to their male counterparts (Table 4). This was consistent with some previous researches (Chong and Chan, 2010 - Korkmaz et al., 2011 - Beyen et al., 2013 - Erick and Smith, 2014). A possible explanation for gender differences in the current study could be attributed to the high percentage of female teachers compared to their male colleagues.
As regards the occupational factors as increasing work duration per year, hours of work per day and working on the computer; were positively associated with increasing risk of CTS among studied teachers (Table 4). This was consistent with previous work done by Cardoso et al., 2009 on the prevalence of musculoskeletal pain among teachers. A similar association has been found in a research work among Indian teachers done by Mariammal et al., 2012 on their study on occupation influenced physical illness observed among the teachers of Thoothukudi town. Conversely, research conducted in Turkey among teachers failed to produce any statistically significant association with teaching experience (Baskurt et al., 2011 - Durmus and Ilhanli, 2012).

\section{Limitations of the study}

A community based study, including asymptomatic teachers was desirable. So this study had a limitation as it was a hospital-based study which limits the actual prevalence of CTS among teachers, but it reflects the prevalence among symptomatic teachers in Menoufia University. Because nerve conduction studies are expensive, it was not possible to take a larger number of participants. Despite this limitation, the present study reflects the association between teach- 
ing profession and its risk factors with CTS in Menoufia University. Therefore, the results should be confirmed and verified by large-scale studies.

Conclusion and recommendations: This study reported a high prevalence of CTS in symptomatic teachers referred to the Physical Medicine, Rheumatology and Rehabilitation department, Menoufia University Hospital, Egypt. The risk factors associated with the prevalence of CTS were female gender, preparatory and secondary school levels, increasing work duration and working hours per day. To decrease the rate of CTS, especially among teachers will require health education, and ergonomic control.

\section{Acknowledgment}

We would like to express our sincere gratitude and deepest appreciation to teachers who accepted to participate in this study.

\section{Conflict of interest}

None

\section{Funding}

None

\section{References}

1. Alsiddiky A, Algethami H, Ahmed E, Tokhtah H and Aldouhan J (2014): The prevalence of musculoskeletal pain \& its associated factors among female Saudi school teachers. Pak J Med Sci; 30(6): 1191-6.
2. American Academy of Orthopaedic Surgeons. Clinical practice guideline on the diagnosis of carpal tunnel syndrome (2007): http://www.aaos.org/Research/guidelines/ CTS_guideline.pdf. (Accessed March 2018).

3. Baskurt F, Baskurt Z and Gelecek N (2011): Prevalence of self-reported musculoskeletal symptoms in teachers. S.D.U. Saglik Bilimleri Enstitusu Dergisi Cilt; 2: 58-64.

4. Beyen TK, Mengestu MY and Zele YT (2013): Low Back Pain and Associated Factors among Teachers in Gondar Town, North Gondar, Amhara Region, Ethiopia. Occup Med Health Aff; 1:5.

5. Boschman J, Frings-Dresen $M$ and Van der Molen H (2015): Use of Ergonomic Measures Related to Musculoskeletal Complaints among primary school teachers: A 2-year Follow-up Study. Saf Health Work; 6(2):90 - 6.

6. Burton C, Chesterton L, Davenport G, Foley M and Silverstein B (2013): Developing agreed clinical criteria for the diagnosis of carpal tunnel syndrome in primary care a clinical consensus exercise. Society of Academic Primary Care Annual Conference . Am J Ind Med; 50(3):155.72-

7. Burton CL, Chesterton LS, Davenport G, Gallo A and Frediani B (2014): Diagnosing and managing carpal tunnel syndrome in primary care. Br J Gen Pract; 64(622):2623.

8. Cardoso JP, De Queiroz I, Maria de Araujo , and Carvalho FM (2009): Prevalence of musculoskeletal pain among teachers. Revista Brasileira de Epidemiologia; 12:1-10. 
9. Cherian A and Kuruvilla A (2006): Electrodiagnostic approach to carpal tunnel syndrome. Technical Note; 9 (3): 177- 82.

10. Chong EY and Chan AH (2010): Subjective health complaints of teachers from primary and secondary schools in Hong Kong. Int J Occup Saf Ergon; 16: 23-39.

11. Durmus D and Ilhanli I (2012): Are there work-related musculoskeletal problems among teachers in Samsun, Turkey? J Back Musculoskelet Rehabil; 25: 5-12.

12. Erick P and Smith D (2014): The prevalence and risk factors for musculoskeletal disorders among school teachers in Botswana. Occup Med Health Aff; 2:4

13. Erick P and Smith D (2015): Musculoskeletal disorders in the teaching profession an emerging workplace hazard with significant repercussions for developing countries. Ind Health; 53(4):385- 6.

14. Graham B, Regehr G, Naglie G and Wright J (2006): Development and validation of diagnostic criteria for carpal tunnel syndrome. J Hand Surg; 31A (6): 919-24.

15. Harris-Adamson C, Eisen E, Kapellusch J, Ghasemi M and Rezaee M et al. (2015): Biomechanical risk factors for carpal tunnel syndrome: a pooled study of 2474 workers. Occup Environ Med; 72(1):33-41.

16. Korkmaz NC, Cavlak U and Telci EA (2011): Musculoskeletal pain, associated risk factors and coping strategies in school teachers. Scientific Research and Essays; 6:649- 57.

17. Lee WJ, Liao YC, Wei SJ, Tsai CW and Chang MH (2011): How to make electro- diagnosis of carpal tunnel syndrome with normal distal conductions? J Clin Neurophysiol; 28:45-50.

18. Mengestu MY (2013): Low Back Pain and Associated Factors among Teachers in Gondar Town, North Gondar, and Amhara Region, Ethiopia. Occup Med Health Aff; 01(05):1-8.

19. Mariammal T, Amutha Jaisheeba A and Sornaraj R (2012): Occupation Influenced Physical Illness Observed Among the Teachers of Thoothukudi Town. Int J Pharm Tech Res; 4:1274- 8.

20. Nirav P and Sanket K (2018): Prevalence of the musculoskeletal disorder among school teachers. Natl J Physiol Pharm Pharmacol; 8(2): 197-201.

21. Shuai J, Yue P, Li L, Liu F and Wang S (2014): Assessing the effects of an educational program for the prevention of workrelated musculoskeletal disorders among school teachers. BMC Public Health; 1:9.

22. Wang YJ and Yan SH (2013): Improvement of diagnostic rate of carpal tunnel syndrome with additional median-to-ulnar comparative nerve conduction studies. Acta Neurol Taiwan; 22:152- 7

23. Werner R (2006): Evaluation of WorkRelated Carpal Tunnel Syndrome. J Occup Rehabil; 16 (2): 201- 16.

24. Yacoub G (2002): Carpal tunnel syndrome: a series observed at Jordan University Hospital (JUH), June 1999-December 2000". Clin Neurol Neurosur; 104 (1): 49-53. 